\title{
Los Dispute Boards en contratos de obra: a propósito de la nueva ley de contrato de participación público privado en Argentina
}

\author{
Francisco J. Muñoz* \\ Recibido/Received: 27/06/2017 \\ Aceptado/Accepted: 21/07/2017
}

SUMARIO: 1. Introducción. 2. Sobre los paneles de expertos. 2.1 Nociones generales de los paneles de expertos. 2.2 Beneficios y ventajas de los paneles de expertos. 2.3 Encuadre de los paneles de expertos en el derecho argentino y en la órbita latinoamericana. 3. Análisis práctico de los paneles de expertos. 3.1 Acerca del nacimiento del panel de expertos. 3.2 Acerca del funcionamiento de los paneles de expertos. 3.3 Acerca del procedimiento de resolución de controversias y las decisiones adoptadas por el panel de expertos. 4. Conclusiones.

PALABRAS CLAVES: Dispute Boards, paneles de expertos, contratos de obra, participación público privada.

KEYWORDS: Dispute Boards, panels of experts, construction contracts, public-private participation.

RESUMEN: Los Dispute Boards son un mecanismo de resolución temprana de controversias, de carácter contractual y

\footnotetext{
Asociado en el equipo de Resolución de Disputas de Rattagan, Macchiavello, Arocena, Buenos Aires, Argentina, Master en Derecho por la New York University (NYU), Profesor de Derecho Internacional Privado de la Universidad del Salvador, Buenos Aires, Argentina.

F. J. MUÑOz, "Los Disputes Boards en contratos de obra: a propósito de la nueva ley de contrato de participación público privado en Argentina”, Revista Ecuatoriana de Arbitraje, No. 8, 2016.
} 
no jurisdiccional, que presentan grandes ventajas en los contratos de obra. Su estudio y entendimiento resulta de vital importancia para comprender cómo los proyectos de gran envergadura pueden mejorarse y ejecutarse en el tiempo estimado. En Argentina, la Ley 27.328 de Contrato de Participación Público Privada, incorporó al ordenamiento legal argentino la figura de los paneles de experto. Consideramos que esta introducción representa un gran espaldarazo al desarrollo de este instituto con la finalidad de implementarlo en grandes proyectos de infraestructura. Proponemos a través de este artículo, el análisis de este instituto, y su validez en el ordenamiento jurídico argentino, como así también brindar al lector con un somero análisis del instituto en la práctica.

ABSTRACT: Dispute Board is an early alternative dispute resolution mechanism mostly used in construction agreements. This mechanism arises from a contractual clause agreed by the parties and issue decisions lacking of jurisdictional powers. The study and correct understanding of these systems are necessary to understand how the execution of major infrastructure projects may be improved and done in time. In Argentina, Act 27.328 (Public-Private Partnership agreements law) incorporated Dispute Boards to the local legal system. In our opinion, said incorporation represents a significant development of this mechanisms towards its implementation in major infrastructure projects. We propose through this article to analyze the mechanism, analyze its validity and legality under Argentine legal system, and provide the lector with an overview of the mechanism in practice.

\section{INTRODUCCIÓN}

Los contratos de obra por medio de los cuales se materializa la realización de grandes proyectos de infraestructura y construcción, presentan grandes desafíos a la hora de su ejecución. Ello 
en virtud que estamos en presencia de un contrato que usualmente es de larga duración y en el que se presentan cambios de escenarios sociales, políticos y económicos durante su ejecución, que en gran medida afectan el normal desarrollo de este contrato.

Por estos motivos, la doctrina ha tendido a buscar instrumentos que permitan a las partes lograr la armonización de sus intereses con miras a lograr el fin último que se tuvo a la hora de contratar: la ejecución de la obra.

El Dispute Board, o panel de experto ${ }^{1}$, ha sido una de estas herramientas que se ha incorporado a diversos proyectos de gran envergadura, con la finalidad de lograr que un panel independiente tome decisiones (con carácter de recomendación o vinculante, de acuerdo al tipo de panel que se opte) a lo largo de la realización de la obra, logrando que se mantenga el cash flow de la misma y evitando que la ejecución se detenga.

Con el convencimiento de que el Dispute Board representa un beneficio significativo para las partes, este sistema se ha incorporado en diversos instrumentos de contratación. El artículo 20 del Contrato FIDIC (International Federation of Consulting Engineers) incorpora una cláusula escalonada previendo como mecanismo de resolución de disputas un Dispute Adjudication Board y luego arbitraje.

Siguiendo con el análisis normativo, la Ley de Contrato de Participación Público Privada argentina, sancionada el 30 de noviembre de 2016, bajo el número 27.328 (Ley de PPP) ${ }^{2}$, ha venido a prever la posibilidad de constituir un panel de expertos para resolver cuestiones de índole técnica que puedan presentarse durante la ejecución del contrato.

Perú, Brasil y Chile también han dado preponderancia a los paneles de expertos para la resolución de controversias de carác-

1. Si bien existen algunas diferencias menores, para el propósito de este trabajo los asimilaremos.

2. Puede consultarse en «https://goo.gl/ghThSH» y el respectivo decreto reglamentario 118/17, que se puede obtener en «https://goo.gl/3fsTx3». 
ter técnico en un contrato de obra. Veremos que estos países han considerado que los Dispute Boards presentan grandes beneficios y por eso extendieron su aplicación hasta en contratos de obra que podrían ser considerados públicos, en algunos casos. Por consiguiente, puede observarse un fenómeno puntual en América Latina en búsqueda de un instrumento que permita sortear obstáculos concretos en la ejecución de obras: corrupción, aumento excesivo de costos, problemas técnicos y freno de la obra como mecanismo de negociación.

El objetivo del presente trabajo es brindar reflexiones sobre los paneles de experto, analizando su concepto, características, beneficios, clases y su análisis práctico. El análisis se efectuará a través de una visión crítica del instituto, tomando en cuenta el reglamento de la Cámara de Comercio Internacional de Dispute Boards (Reglamento de Dispute Boards de la CCI) y analizando la Ley de PPP y el tratamiento dado por diversos países latinoamericanos a esta cuestión.

\section{SOBRE LOS PANELES DE EXPERTOS}

Los paneles de expertos fueron definidos como un sistema de resolución de disputas, de naturaleza contractual, en donde terceros expertos independientes e imparciales, entienden en controversias fácticas de manera temprana, brindando recomendaciones o resoluciones de carácter vinculante ${ }^{3}$.

A continuación, desarrollaremos un análisis de la definición de los paneles de expertos (2.1.), sus principales ventajas en contratos de obra (2.2.) y su encuadramiento en el derecho latinoamericano, con especial énfasis en el derecho argentino (2.3.).

3. Para mayor precisión, véase, las definiciones de Dispute Board adoptadas por la Dispute Resolution Board Foundation y la CCI en sus respectivos reglamentos. 


\subsection{Nociones generales sobre los paneles de expertos}

Tal como hemos definido anteriormente, los paneles de expertos son efectivamente mecanismos de resolución de controversias. Existe un panel de expertos, independientes e imparciales, que emiten sus decisiones ante las controversias que las partes les presentan.

a. Observamos en primer lugar que el surgimiento de los paneles de expertos se da por la incorporación de este instituto a un contrato determinado ${ }^{4}$. Las partes deberán incluir la referencia expresa a sujetar ciertas controversias a los paneles de expertos.

Decimos ciertas controversias, ya que en general, solamente disputas de carácter fáctico o técnico son sometidas a los paneles de expertos.

Los paneles de expertos brindan una solución (con carácter de recomendación o también puede ser vinculante), de manera efectiva y en tiempo real ${ }^{5}$. Es decir, los paneles de expertos emiten sus decisiones en un período de tiempo corto, que posibilita la continuación de la obra sin que sea necesaria su detención a las resultas de la decisión judicial o arbitral.

Destacamos también, en base a los principales reglamentos de Dispute Boards, que las decisiones emitidas por los paneles de expertos no son de carácter jurisdiccional ${ }^{6}$. Esta mención no es solamente teórica, sino que tiene efectos prácticos. Las decisiones de los paneles de expertos pueden ser revisadas por la autoridad jurisdiccional elegida, sin que se hubiera precluido el tratamiento de la controversia.

4. C. Chern, Chern on Dispute Boards, 3ra Ed., Informa Law from Routledge, 2015, pp. 42-43. El autor sostiene que los paneles de expertos nacen de contratos ya que las partes otorgan a los paneles de expertos ciertas facultades jurisdiccionales para investigar un reclamo o disputa y también facultades para decidir dichos reclamos o disputas.

5. Véase, el Prefacio del Reglamento de Dispute Boards de la CCI.

6. Véase a modo de ejemplo, el Art. 1 del Reglamento de Dispute Boards de la CCI. 
b. En segundo lugar, debemos mencionar que los paneles de expertos pueden constituirse al momento del perfeccionamiento de la relación contractual y participando de toda la ejecución del proyecto, o puede constituirse ad hoc, es decir, frente al acaecimiento de una disputa determinada.

La práctica habitual indica que las partes prefieren la constitución de los paneles de expertos conjuntamente con el perfeccionamiento del contrato ${ }^{7}$. Ello debido a que: (i) los paneles de expertos tienen pleno conocimiento del proyecto desde su inicio; (ii) los miembros de los paneles están informados de los principales sucesos durante la ejecución del contrato, teniendo una visión más acabada del proyecto; y (iii) los paneles podrán ejercer también funciones preventivas al estar capacitados para detectar eventuales problemas que puedan surgir ${ }^{8}$.

c. En tercer lugar, es importante destacar el tipo de decisión que podrán adoptar los paneles de expertos. En este sentido, clásicamente se ha entendido que los paneles de expertos pueden emitir recomendaciones, decisiones con carácter vinculante, o ambas.

Estas recomendaciones o resoluciones vinculantes que emiten los Dispute Boards pueden formar parte del contrato, en la medida en que las partes así lo acuerden. Si es el caso que las decisiones de los paneles de expertos forman parte del contrato, su incumplimiento, por cualquiera de las partes, da origen a un incumplimiento contractual ${ }^{9}$.

La determinación del carácter de la decisión del panel de expertos dará lugar a la determinación del tipo de panel con el que estaremos lidiando.

7. G. Owen y B. TotTerdill, Dispute boards: procedures and practice, 1ra. Ed., Thomas Telford, 2008, pp. 4-7.

8. La utilización de los paneles de expertos no se limita exclusivamente a casos de contratos de obra. Véase, C. Koch, "The New Dispute Board Rules of the ICC", 23 ASA Bulletin 1/2005 (Mars), p. 56.

9. Para una definición y mayor detalle de cada tipo de DB, véase G. J. VAZ y P. A. GraVATÁ Nicoli, "Los Dispute Boards y los contratos administrativos: ¿Son los DB's una buena solución para disputas sujetas a normas de orden público?", en R. HERnÁNDEZ GARCía (Coor.), Dispute Boards en Latinoamérica: experiencia y retos, Biblioteca de Arbitraje del Estudio Mario Castillo Freyre, Vol. 23, p. 100. 
En este sentido, se ha reconocido que el Dispute Review Board es aquel que resuelve disputas por medio de recomendaciones ${ }^{10}$. Así, si bien no serán vinculantes para las partes, la decisión del panel generará una fuerte convicción respecto de la necesidad de cumplir con dicha resolución caso contrario, se perderá credibilidad frente al panel de expertos. Estas recomendaciones también podrán transformarse en decisiones vinculantes si el reglamento elegido por las partes así lo dispone ${ }^{11}$.

Esta forma de panel de expertos es la adoptada por la Ley de PPP, que en su decreto reglamentario 118/17 señaló que se emitirán recomendaciones, que solamente pasarán a ser vinculantes siempre y cuando las partes no la impugnen en el plazo y conforme la vía estipulados.

Los Dispute Adjudication Boards son aquellos que emiten resoluciones vinculantes para las partes ${ }^{12}$.

Este tipo de decisiones deben ser cumplidas por las partes bajo pena de acarrear sanciones. Sin perjuicio de que las decisiones pueden ser revisadas por vía judicial o arbitral (dependiendo de la jurisdicción pactada por las partes), la parte ganadora podrá ejecutar la decisión en cualquier momento.

Los contratos FIDIC han optado por este tipo de panel de expertos.

Finalmente, nos encontramos con los Combined Dispute Boards, en los cuales los miembros del panel podrán emitir recomendaciones o resoluciones vinculantes de acuerdo al caso y a lo pactado expresamente por las partes.

10. C. A. Peñate Guzmán, "Las mesas de resolución de disputas como mecanismo que viabiliza la continuidad de los proyectos de construcción: la experiencia en El Salvador", en R. HernÁNDEZ GARCía (Coor.), Dispute Boards en Latinoamérica: experiencia y retos, Biblioteca de Arbitraje del Estudio Mario Castillo Freyre, Vol. 23, p. 89.

11. Para una definición y mayor detalle de cada tipo de DB véase, G. J. VaZ y P. A. Gravatá Nicoli, pp. 111-112.

12. C. A. Peñate Guzmán, N. 11, p. 89. 
d. Tal como hemos dicho, los paneles de expertos estarán regidos por lo acordado por las partes, y en particular, por el reglamento elegido por ellas.

En la práctica observamos distintos tipos de reglamentos, entre los que destacamos: el Reglamento de Dispute Boards de la CCI mencionado anteriormente, y el Reglamento de Dispute Boards de la Dispute Resolution Board Foundation.

No se nos escapa el hecho de que las partes pueden pactar sus propias reglas. Sin embargo, consideramos que sería extremadamente dificultoso el correcto andar del panel en caso de un sistema ad hoc dado que todavía no se está habituado a este instituto. Ello, más allá de las complejidades propias que presenta el optar por redactar las normas de un sistema de resolución de disputas, tal como está probado en el arbitraje.

Ahora bien, no es necesario destacar lo fundamental que resulta tener un reglamento, pero si nos gustaría resaltar que la forma de nombrar a los miembros del panel, el procedimiento a seguir para resolver una controversia, la admisibilidad de pruebas, la clase de decisión a adoptar, la ejecución de la decisión y su eventual recurribilidad dependerán del reglamento que se opte.

Dentro de los reglamentos comúnmente utilizados encontramos el Reglamento de la Dispute Resolution Board Foundation ${ }^{13}$, institución que preparó una guía en el año 2007 para que sea usada en contratos de obra. La Dispute Resolution Board Foundation es la institución que más promueve este mecanismo de resolución de controversias a nivel mundial, enfocado principalmente a contratos de obra.

También mencionamos el Reglamento de Dispute Resolution de la CCI aprobado en octubre de 2015, que cuenta con treinta y cinco artículos en los que reconoce los distintos tipos de Dispute Boards, los procedimientos a seguir, los costos, entre otras cues13. Para mayor información se puede consultar, «https://goo.gl/DVnTuR». 
tiones. Este reglamento es aplicable solamente si las partes así lo decidan ${ }^{14}$.

e. Nos gustaría analizar una cuestión delicada que está relacionada con la vinculación entre el panel de expertos y el mecanismo de resolución de controversias jurisdiccional pactado por las partes.

En este sentido, es usual encontrar en la práctica cláusulas escalonadas en las que se prevé en un primer lugar la resolución de la controversia por medio del panel de expertos y luego la remisión a arbitraje para el caso que no pudiera ser resuelta o para recurrir la decisión del panel.

Esta cláusula presenta el siguiente interrogante: ¿es un requisito necesario agotar la vía del panel de experto para luego acudir a la sede arbitral o judicial pactada?

Frente a esta situación se plantean varias cuestiones a analizar.

En primer lugar, deberá observarse si de la redacción de la cláusula se deriva que el panel de expertos es un requisito necesario para ir a la sede jurisdiccional pactada. Si ese es el caso, habrá que analizar si el paso previo es un requisito de admisibilidad del reclamo posterior o de jurisdicción.

Como principio general, la mayoría de los reglamentos que prevén cláusulas escalonadas determinan que el panel de expertos es un requisito de admisibilidad necesario para acudir a la jurisdicción ulterior. Tal es el caso de la Ley de $\mathrm{PPP}^{15}$ y de la jurisprudencia comparada. La Corte Suprema de Suiza, en el caso $4^{a}$ 124/2014, resuelto el 7 de julio de 2014, determinó que la cláusula de resolución por Dispute Board incorporada en un contrato FIDIC era un paso previo obligatorio para iniciar el arbitraje.

14. Para mayor información se puede consultar, «https://goo.gl/7esQyz».

15. Véase, Art. 9.13.n) del Decreto Reglamentario 118/17. 
Ahora bien, esta interpretación nos lleva a pensar qué sucede para el caso que la contraparte se niegue a constituir el panel de expertos, o su participación sea determinante para la mentada constitución.

Consideramos que, si la parte que busca el remedio jurisdiccional ha efectuado todos los pasos necesarios para buscar la constitución del panel, y no ha podido efectuarlo, no puede prohibirse acudir a la sede jurisdiccional pactada. Tampoco podría interponerse una excepción de admisibilidad en el arbitraje para el caso que el panel de expertos no hubiera resuelto la controversia, si es que dicha controversia no puede ser sometida a resolución del Dispute Board.

f. Finalmente, nos gustaría dar algunos ejemplos en los que el panel de expertos, o Dispute Board ha resultado ser un aporte significativo a la ejecución del contrato, disminuyendo los reclamos, los costos de la obra y la corrupción.

Tal vez el ejemplo más relevante y quien más incentiva el uso de este instituto es el Banco Mundial ${ }^{16}$, que impone como requisito para el otorgamiento de créditos la utilización de esta clase de mecanismos. Los contratos FIDIC también incluyen paneles de expertos en la cláusula 20.

Las obras más relevantes y millonarias en las que se han utilizado estos paneles son: (i) el proyecto del Túnel en el Canal de la Mancha; (ii) el Aeropuerto de Hong Kong; (iii) el proyecto Hidroeléctrico de Ertan en China; (iv) el Túnel Eisenhower en Estados Unidos; y (v) proyectos de construcción en el marco de los Juegos Olímpicos de Londres 2012 y Río $2016^{17}$.

16. El Banco Mundial incorporó a principios de 1990 Dispute Review Boards a los contratos FIDIC modificados que utilizaba el Banco Mundial y que eran publicados bajo el título Procurement of Works. En el año 2000, el Banco Mundial elaboró una nueva edición de Procurement of Works donde incorporó los Dispute Adjudication Boards. Luego, FIDIC y el Banco Mundial. Para mayor información véase, C. CHern, N. 5, pp. 12 y ss.

17. Véase, J. E. Figueroa VAldÉs, "Dispute Boards: la visión de las partes y su co-existencia con los contratos administrativos. Experiencias en Chile", en R. HERnÁndez GarCía (Coor.), Dispute Boards en Latinoamérica: experiencia y retos, Biblioteca de Arbitraje del Estudio Mario Castillo Freyre, Vol. 23, p. 125. 
En Latinoamérica, además de la utilización para los Juegos Olímpicos de Río, el sistema se utilizó en la expansión de la represa Yacyretá-Apipé en los años 2003-2006 (Argentina), para la construcción de la ampliación del Canal de Panamá, y en los casos de contrataciones del Estado surgidas de la Ley Peruana 30.225, solo por nombrar algunos ejemplos.

\subsection{Beneficios y ventajas de los paneles de expertos}

Hemos indicado algunos de los beneficios más significativos del Dispute Board, como la disminución de la corrupción, de los costos para resolver los conflictos y de la celeridad para entender en las controversias evitando que la obra se detenga.

Sin perjuicio de ello, nos gustaría poner de resalto otros beneficios adicionales, muy significativos también, que demuestran que el panel de expertos es muy recomendable para contratos de obra $^{18}$.

Los beneficios o ventajas de los paneles de expertos radican en:

a. La disminución de los reclamos dado que los paneles de expertos disminuyen los reclamos porque éstos incentivan la comunicación entre las partes, la cooperación y las relaciones positivas para resolver amigablemente las discusiones. Asimismo, los paneles de expertos actúan preventivamente cuando detectan potenciales conflictos emitiendo recomendaciones teniendo siempre como fin último que la obra se lleve a cabo conforme se prevé en el pliego de bases y condiciones.

b. Para el caso de los paneles de expertos que se constituyan desde la firma del contrato, sus miembros estarán en comunicación directa con las partes y conocerán al detalle los avances del proyecto. Ello permitirá detectar problemas, y resolver con agilidad.

18. Interesante análisis efectúa CHERN en su libro respecto de las razones por las cuales debería elegirse DB por sobre otros mecanismos de resolución de conflictos. Véase, C. CHERN N. 5, pp. 26 y ss. 
c. Los miembros del panel de expertos toman decisiones en forma oportuna y en tiempo real, en virtud de su conocimiento del proyecto. Ello trae como consecuencia que el plazo del contrato no se vea mayormente afectado y evita que se disparen los costos del contrato.

d. Los paneles de expertos permiten la resolución ágil de una controversia durante la ejecución del contrato. Ello hace que no sea necesario acudir a la sede jurisdiccional pactada, con el tiempo que conlleva obtener una decisión y la afectación que sufre la obra. Además, los paneles previenen que los pequeños conflictos avancen generando una controversia mayor.

e. Los paneles de expertos disminuyen futuros reclamos frívolos, ya que será más dificultoso para las partes poder construir un caso basado en reclamos inexistentes sobre los cuales el panel de expertos nunca se hubiera expedido.

f. Los paneles de expertos funcionan como una barrera para las medidas de reclamo arbitrarias que puede tomar un constructor, frenando la ejecución de la obra o reclamando sobrecostos.

g. Los paneles de expertos funcionan como un mecanismo de control de la transparencia del proyecto. Siendo que los paneles son parte de las comunicaciones y de los avances del proyecto, evitan corrupción a través de sobrecostos o pagos irregulares.

h. Los paneles de expertos aseguran el mantenimiento del cash flow ya que resolverán reclamos sin demoras.

i. Los paneles de expertos evitan interrupciones, suspensiones o dilaciones en la construcción motivados por la necesidad de aguardar por una resolución judicial.

j. Someter las disputas a un panel de expertos resultará menos costoso para las partes, que acudir a sede judicial o arbitral. 
k. La existencia de un panel de expertos, y la resolución de una controversia por parte de un panel, facilita la producción de prueba. Ello en virtud de que la controversia será sometida al panel a poco tiempo de haber ocurrido, siendo más fácil para las partes producir esos documentos.

1. Las partes pueden adaptar la estructura de los paneles de expertos a sus necesidades y las necesidades del proyecto.

Pese a todas las ventajas enunciadas, un factor será determinante para que el panel de expertos pueda ser útil: la colaboración de las partes ${ }^{19}$. Si las partes no prestan su colaboración y actúan de buena fe, la eficacia y buen funcionamiento del panel estará seriamente comprometida. Veremos que varios reglamentos y la Ley de PPP han incorporado este principio, en particular, para el momento de producir pruebas y celebrar audiencias.

Otra cuestión a destacar para la correcta actuación de los paneles de expertos es la adecuada previsión de los costos que tiene este instituto. Las partes deberán tener presente al momento de celebrar el contrato que el panel de expertos devenga costos concretos, tales como: (i) honorarios de los miembros; (ii) viáticos por viajes a la obra; (iii) gastos por audiencias; (iv) honorarios de la organización que administre el panel, entre otros.

Ahora bien, teniendo en cuenta todos estos rubros, el impacto del panel de expertos en el costo total del proyecto asciende a aproximadamente 0,015 al 0,045\% del total involucrado ${ }^{20}$.

Como se puede apreciar, los costos derivados del funcionamiento del panel no deberían ser un factor decisivo para decidir su inclusión. 


\subsection{Encuadre de los paneles de expertos en el derecho argentino y en la órbita latinoamericana}

Ya hemos brindado un análisis por medio del cual concluimos que los paneles de expertos resultan ser un instituto muy recomendable para incluir en contratos de obra, si es que las partes están verdaderamente interesadas en que el proyecto se lleve a cabo en tiempo y forma.

Ahora nos toca afrontar el desafío de justificar por qué los paneles de expertos son válidos a la luz del derecho argentino, con base en principios que pueden ser extrapolados a otros ordenamientos jurídicos latinoamericanos.

También nos referiremos algunas previsiones del derecho peruano y del derecho brasilero que nos permiten concluir que el panel de expertos es válido en dichas jurisdicciones.

a. Partimos de la base de que los paneles de expertos son válidos a la luz del derecho argentino ${ }^{21}$.

No hay ninguna duda respecto de que los paneles de expertos se adecuan a las previsiones del derecho privado argentino, $y$ por ende, son plenamente válidos para obras privadas.

También consideramos que es posible utilizar paneles de expertos en contratos de obra pública, aunque esta conclusión puede llegar a ser controversial y sujeta a críticas.

b. Los paneles de expertos son válidos a la luz del derecho privado argentino. Diversas razones nos guían a esta conclusión: (i) la nueva regulación de la Ley de PPP que expresamente autoriza los paneles de expertos; (ii) la autonomía de la voluntad de

21. D. Jiménez Figueres y R. Caivano, "Funcionamiento de los 'Dispute Boards' de la CCI y su encuadre en el derecho argentino", Revista Internacional de Arbitraje, N 6, Enero-Junio, Editorial Legis, 2007. Estos autores han abordado la compatibilidad del DB con el derecho argentino, pero con el Código Civil y con el Código de Comercio. Creemos que las conclusiones mencionadas en su artículo son igualmente aplicables al análisis del DB y el Código Civil y Comercial de la Nación (Argentina), en tanto no han habido modificaciones sustanciales respecto la materia disponible en el nuevo ordenamiento jurídico. 
las partes para determinar el contenido de un contrato; (iii) la autorización expresa para prorrogar jurisdicción; y (iv) regulación de institutos análogos en el derecho argentino.

El primer argumento que nos permite arribar a la conclusión indicada anteriormente es la novel previsión de la Ley de PPP introduciendo el panel de expertos. Si bien el contrato de PPP no debería ser calificado como contrato privado, consideramos que la incorporación en un sistema tan rígido como es la contratación pública, debe extenderse necesariamente al derecho privado.

Esta última afirmación encuentra sustento principalmente en el segundo argumento por el que consideramos que los Dispute Boards son válidos en el derecho privado argentino: la autonomía de la voluntad. Así, bajo derecho argentino, las partes son libres de pactar el contenido y alcance de las previsiones contractuales, con el límite de la moral, las buenas costumbres y el orden público. Al reconocerse el principio de autonomía de la voluntad material ${ }^{22}$, las partes se encuentran habilitadas para pactar los términos contractuales que ellas consideren adecuadas, que pueden incluir, entre otras cuestiones, mecanismos de resolución de controversias de carácter contractual (como es un Dispute Board).

No quedan dudas entonces que, bajo el principio de la autonomía de la voluntad, los paneles de expertos son válidos ya que su incorporación en nada afecta la moral, las buenas costumbres ni el orden público.

A mayor abundamiento, analizando los principios particulares regulados para el contrato de obra de carácter privado, encontramos que el artículo 1253 del Código Civil y Comercial de la Nación faculta a las partes a determinar los medios más adecuados para la correcta ejecución del contrato. Como hemos indicado, al no ser los paneles de expertos un mecanismo de resolución de controversias de carácter jurisdiccional, puede comprenderse como un mecanismo que facilita y colabora con la más eficaz ejecución del contrato, encuadrándose entonces dentro de 22. Véase, Art. 958 del Código Civil y Comercial de la Nación (Argentina). 
los fines perseguidos por el legislador al redactar el mentado artículo.

En tercer lugar, ponemos sobre la mesa un argumento de razonabilidad. Si las partes están facultadas a prorrogar jurisdicción a favor de otros tribunales (judiciales o arbitrales) de carácter jurisdiccional, también deberían estar facultadas para elegir un mecanismo de resolución de controversias que no emite decisiones jurisdiccionales.

Este argumento se ve reafirmado también por el hecho de que el tribunal que deba entender en la controversia (ya sea por elección de las partes como por aplicación de las normas procesales para determinar la jurisdicción), podrá con posterioridad abrir, inspeccionar, examinar y revisar completamente la decisión del panel de expertos, y evaluar los daños que hubiera podido ocasionar la resolución adoptada por dicho panel.

En cuarto lugar, si bien el ordenamiento vigente (a excepción de la Ley de PPP) no contiene ninguna referencia expresa acerca de la validez de los paneles de expertos, sí existen mecanismos de resolución de controversias similares a los Disputes Boards como ser los peritos árbitros ${ }^{23}$.

Finalmente, la Ley de PPP expresamente incorporó en el artículo 9 inciso w) este instituto. Dicho artículo establece que:

Sin perjuicio de lo que se establezca en la reglamentación, en los pliegos y en la documentación contractual, los contratos de participación público-privada deberán contener las siguientes previsiones: $\mathrm{w}$ ) los procedimientos y métodos que resultarán de aplicación para dirimir las controversias de índole técnica, interpretativa o patrimonial que puedan suscitarse durante la ejecución y terminación del contrato. A estos efectos, podrá constituirse un panel técnico a partir de la entrada en vigencia del contrato, integrado por profesionales y/o representantes de universidades nacionales o extranjeras, en todos los casos, de acreditada independencia, imparciali-

23. Véase, Art. 773 del Código Procesal Civil y Comercial de la Nación (Argentina). 
dad, idoneidad y trayectoria nacional e internacional en la materia, el que subsistirá durante todo el período de ejecución para dilucidar las cuestiones de tal naturaleza que se susciten entre las partes.

El artículo 9, punto 13 del decreto reglamentario de la Ley de $\mathrm{PPP}^{24}$, ha tratado en detalle los paneles de expertos que pueden constituirse en el marco de los contratos PPP. En cada punto que analizaremos a continuación, haremos una breve mención de lo establecido en este reglamento.

c. Creemos también que es necesario poner de resalto que otros países de Latinoamérica ya han tenido la oportunidad de expedirse respecto de la viabilidad de los paneles de expertos bajo el derecho de dichos países. Observamos en la actualidad una corriente favorable para la inclusión de este sistema dentro de los contratos de obra.

A modo de ejemplo, nos gustaría referirnos a la situación del derecho peruano. Los paneles de expertos en Perú están siendo muy utilizados, principalmente desde la reforma de la Ley de Contrataciones del Estado, efectuada mediante la ley 30.225. En dicha reforma, se ha incorporado al panel de expertos como un medio de resolución de controversias durante la ejecución contractual ${ }^{25}$.

Como podemos apreciar, Perú ha avanzado enormemente en su consideración respecto de los paneles de expertos, ya que ha incorporado la posibilidad de pactarlos para contratos de obra pública. Creemos que este gran avance en la materia, como así también la experiencia peruana que podrá verse en la práctica, demostrará a los países más reticentes, que el panel de expertos sería una gran opción para contratos de obra pública.

Brasil es otro caso a analizar, ya que recientemente ha sido reconocido por el Consejo de Justicia de Brasil, que el panel de

24. Decreto Reglamentario 118/17.

25. Véase, «https://goo.gl/SMuoiZ». 
expertos es un instituto efectivo, y recomendó a los tribunales inferiores incentivar su utilización ${ }^{26}$. El Estado de Río de Janeiro ha permitido que, en el marco de los Juegos Olímpicos celebrados en 2016, se pudieran prever paneles de expertos en ciertos contratos.

\section{ANÁlisis PRÁCTICO de los PANELES DE EXPERTOS}

Anteriormente, hemos intentado brindar un análisis respecto de los puntos teóricos centrales del panel de experto, concentrándonos particularmente en tratar de explicar su concepto, ventajas, y encuadre jurídico en el derecho civil.

Ahora bien, en este apartado buscaremos responder cómo funciona un panel de expertos en la práctica. Para ello, consideramos que lo mejor es partir desde la génesis de un panel de expertos, que será la cláusula negociada por las partes e incluida en el contrato. Ello nos permitirá abordar luego las cuestiones derivadas del funcionamiento del panel hasta la resolución de la controversia.

\subsection{Acerca del nacimiento del panel de expertos}

No puede responderse el interrogante respecto de cómo funciona un panel de expertos sin antes analizar cómo surge el panel y la importancia que tienen las previsiones contractuales que las partes pudieran hacer a la hora de celebrar el contrato de obra.

No hay discusión respecto del carácter contractual del nacimiento de los Dispute Boards. Los paneles de expertos surgen por la autonomía de la voluntad de las partes, que, a la hora de contratar, decidieron incluir este sistema de resolución temprana de disputas como una cláusula más del contrato.

26. J. C. Bueno y A. Figueiredo, "It's all about the Project! - Dispute Boards are recognized by Brazil's Council of Federal Justice", Dispute Resolution Board Foundation newspaper, 01/09/16. 
Por ello, consideramos que para el verdadero éxito de un panel de expertos resulta imprescindible la concreta y correcta redacción de una cláusula de Dispute Board, la elección del tipo de decisión que el panel emitirá, el reglamento aplicable y su interacción con los tribunales jurisdiccionales.

Al igual que lo que sucede con las cláusulas arbitrales, instituciones internacionales han redactado cláusulas modelo que las partes pueden adoptar e incorporar a sus contratos. El Reglamento de Dispute Boards de la CCI brinda la siguiente cláusula modelo:

Por la presente, las Partes se comprometen a constituir un Dispute Adjudication Board (DAB) conforme al Reglamento de la Cámara de Comercio Internacional (CCI) relativo a los Dispute Boards (el Reglamento), el cual es considerado parte integrante del presente. El DAB se compone de (uno/tres/ $x$ ) miembro(s) nombrado(s) en el presente contrato o nombrado(s) de acuerdo con el Reglamento.

Todas las controversias derivadas del presente contrato o relacionadas con él serán sometidas, en primer lugar, al DAB conforme al Reglamento. Para cualquier controversia, el DAB dictará una Decisión de conformidad con el Reglamento.

Si una de las Partes no acata una Decisión cuando se le exija hacerlo de conformidad con el Reglamento, la otra Parte puede, sin tener que recurrir primero al $\mathrm{DAB}$, someter este incumplimiento a arbitraje de acuerdo con el Reglamento de Arbitraje de la CCI, a uno o más árbitros nombrados conforme a este Reglamento de Arbitraje. Una Parte que no cumpla con una Decisión cuando se le exija hacerlo de conformidad con el Reglamento no podrá plantear ningún asunto sobre el fondo de la Decisión como defensa de su falta de cumplimiento sin demora de la Decisión.

Si una Parte notifica por escrito a la otra y al DAB su desacuerdo con una Decisión, según lo previsto en el Reglamento, o bien si el DAB no ha dictado una Decisión en el plazo previsto por el Reglamento, o incluso si el DAB es disuelto conforme al Reglamento antes de que se dicte la Decisión, la controversia será resuelta definitivamente de acuerdo con el Reglamento de Arbitraje de la CCI por uno o más árbitros nombrados conforme a este Reglamento de Arbitraje. 
Finalmente, los redactores de las cláusulas deberán tener presente los alcances del reglamento, de las funciones de los miembros del panel, y de las atribuciones del panel de expertos. Ello, con la finalidad de que este panel pueda ser lo más eficiente posible para cumplir con el objetivo de lograr soluciones de disputas de manera veloz y temprana.

En nuestro criterio, y dado el poco desarrollo que ha tenido este instituto en nuestra región, lo más aconsejable sería redactar una cláusula de Dispute Board tomando como referencia las cláusulas modelos propuestas por las distintas organizaciones, y adoptar también un reglamento aplicable que hubiera sido suficientemente probado.

\subsection{Acerca del funcionamiento de los paneles de expertos}

Ya hemos indicado que el panel de expertos se regirá de acuerdo a los términos de la cláusula y, en particular, del reglamento elegido. Para el caso del panel de expertos que surgirá de los contratos PPP en el marco de la Ley de PPP de Argentina, también deberán considerarse la mentada ley y su decreto reglamentario, como fuentes que regirán el panel.

a. La constitución del panel es el punto de partida del funcionamiento del mismo. Para ello, deberán observarse las formas determinadas por las fuentes aplicables para entender cómo se nombrarán los miembros, las posibilidades de remoción o recusación de los integrantes, como así también el momento en el cual deberán constituirse.

En relación a la constitución, en general los reglamentos internacionales en la materia determinan que el panel esté compuesto por tres miembros (dos ingenieros y un abogado es la composición habitual). El decreto reglamentario 118/17 de la Ley de PPP determina que el panel estará compuesto de cinco miembros $^{27}$. Entendemos que esta norma puede ser dejada de lado por

27. Véase, Art. 9.13.b) del Decreto Reglamentario 118/17. 
las partes y pactar un panel de tres miembros que será menos costoso.

El nombramiento por lo general es de común acuerdo, con la expectativa de que las partes puedan lograr un consenso respecto de la idoneidad y legitimidad de los miembros que regirán la ejecución del contrato. A diferencia del arbitraje, la intención es que las partes puedan acordar los miembros en común, ya que no se tiene como objetivo la protección de los intereses de cada una de las partes, sino la correcta ejecución de un proyecto determinado. En otras palabras, se busca proteger el desarrollo adecuado del proyecto.

En general, los miembros del panel surgen de un listado elaborado por el ente administrador. A modo de ejemplo, para el caso de los paneles de expertos que surgen de la Ley de PPP, el listado surgirá de un concurso público efectuado por la Unidad de Participación Público Privada.

Para el caso que no existiera acuerdo, el panel será elegido por el organismo administrador del panel de expertos. La Unidad de Participación Público Privada también será la encargada de elegir los miembros del panel si no hubiera acuerdo entre las partes.

Para el caso que una de las partes se mostrara renuente a elegir a los miembros, la otra parte podrá requerir la constitución a la entidad administradora ${ }^{28}$.

Una vez elegido el panel, se generará entre las partes y los miembros una relación contractual. Expresamente se ha previsto que las partes firmen un acuerdo para regular las obligaciones, los honorarios, la forma de trabajar, entre otras cuestiones ${ }^{29}$.

Ante este escenario (el de la relación contractual entre los miembros de los Dispute Boards y las partes del contrato) surgen

28. Así lo prevé expresamente el Art. 7 del Reglamento de Dispute Boards de la CCI.

29. Véase a modo de ejemplo, el Art. 10 del Reglamento de Dispute Boards de la CCI. 
diversas consideraciones que deben tenerse en cuenta a la hora de pensar en la incorporación de los Dispute Boards como mecanismo previo de resolución de conflictos: (i) clase de responsabilidad en caso de incumplimiento de las obligaciones de cualquiera de las partes; (ii) forma de reclamar el cumplimiento de las obligaciones asumidas; y (iii) ¿existen mecanismos de terminación del contrato a favor de los miembros de los Dispute Boards por incumplimiento de las partes?

b. Los miembros elegidos para integrar el panel deberán ser y permanecer independientes e imparciales. Estas obligaciones nacen de los reglamentos aplicables, que expresamente la prevén, como así también de la Ley de PPP que estableció esta obligación. No resulta extraña esta obligación, ya que es común que cualquier impartidor de justicia, o tercero que participa en la resolución de un conflicto deba ser y mantenerse ajeno a las partes.

Decimos que deberán ser ya que al momento de ser elegidos no puede dudarse respecto de su independencia e imparcialidad. Y decimos que deberán permanecer, ya que esta obligación estará vigente a lo largo de toda la ejecución del contrato.

Las obligaciones de independencia e imparcialidad son pilares fundamentales que hacen a la credibilidad de los miembros. Ambas obligaciones están orientadas a buscar garantías de que la decisión que va a tomar el panel no beneficiará a ninguna de las partes intencionalmente.

c. Otro aspecto a considerar, que hace también a la legitimidad de los miembros del panel, es la posibilidad de requerir la recusación de los miembros. Las causales de recusación están previstas en los reglamentos de paneles de expertos.

En este sentido, el Reglamento de Dispute Boards de la CCI lista las causales de recusación y remoción de los miembros, y su proceso de sustitución. Los artículos 8.4 y 8.5 determinan el proceso de recusación y remoción de los miembros por falta de imparcialidad, independencia, y bajo la frase "cualquier otro 
motivo" deja a salvo otras circunstancias que podrían afectar una resolución justa si se mantiene esa persona como experto.

Brevemente, el proceso de sustitución de un experto consistirá en la presentación de las razones por las cuales debe ser sustituido (muerte, incapacidad, o recusación). Luego, se nombrará de acuerdo al procedimiento determinado en el contrato o en el reglamento (que fuera explicado anteriormente). En el caso de sustitución por recusación, del pedido de sustitución se ordena el traslado al miembro acusado, a los restantes miembros y a la otra parte, para que brinden su opinión al respecto. Luego, la CCI decidirá sobre la procedencia de la recusación ${ }^{30}$.

\subsection{Acerca del procedimiento de resolución de controversias y las decisiones adoptadas por el panel de expertos}

Una vez constituido el panel de expertos, el mismo comenzará a operar, siempre teniendo en miras la correcta ejecución del proyecto y detectando los conflictos que pudieran presentarse. Para el caso que el panel se constituyera a los fines de resolver una controversia puntual, se atenderá dicho conflicto, y luego participarán activamente en la ejecución de la obra.

Ahora bien, resulta interesante analizar cómo los miembros del panel llegan a emitir la recomendación o la decisión vinculante respecto de un tema. Para ello, existe un procedimiento, que es el que analizaremos a continuación, con el fin de regular el correcto ejercicio del derecho de defensa y petición de las partes, que nunca puede ser ignorado por el panel, pese a no ser una decisión con carácter jurisdiccional.

Es usual que el procedimiento conste de tres etapas bien diferenciadas, al igual que un proceso jurisdiccional (tanto frente a un tribunal judicial como arbitral). En primer lugar, encontramos la etapa de presentación del reclamo frente al panel. En segundo lugar, se halla la etapa de prueba o audiencia. En tercer lugar, la etapa de resolución de la controversia planteada.

30. Véase, Arts. 7 y 8 del Reglamento de Dispute Boards de la CCI. 
Como hemos dicho, las etapas en un procedimiento de Dispute Board son conceptualmente similares a las de un procedimiento judicial o arbitral. Sin embargo, en la práctica, existen diferencias sustanciales que veremos cuando analicemos cada una de estas etapas.

a. En la etapa inicial ${ }^{31}$, la parte peticionante (que puede ser el contratista o el comitente, de acuerdo al reclamo) presentará una demanda ante el panel de expertos, explicando y fundando su reclamo. Por lo general, se acompañan constancias documentales del reclamo, como así también otra prueba a producirse, y eventuales propuestas de solución.

Recuérdese, las controversias son de carácter técnico y por eso es necesario que se acompañen las constancias o pruebas documentales que acrediten la falla técnica que se busca reparar.

El panel técnico oirá a la otra parte, corriéndole traslado de la demanda presentada por el peticionante. También se le permitirá a la contraparte acompañar los documentos y ofrecer las pruebas conducentes.

Esta primera etapa generalmente es muy breve. Los escritos no son grandes demandas como las que se acostumbran a ver en arbitrajes de construcción, ya que es usual que solo involucre un tema técnico puntual. Asimismo, los plazos de traslado para que el demandado siente su posición son muy breves también. Todo con el ánimo de obtener una resolución expedita que no demore la ejecución del proyecto.

El Reglamento de Dispute Boards de la CCI va en este sentido. El procedimiento se inicia con la petición formal de requerimiento de iniciación de proceso de "Desavenencia". Este escrito contendrá un relato de los hechos que dan origen a la controversia, las partes involucradas, la lista de puntos que deben ser resueltos por el panel, medidas cautelares o urgentes (si es que fuera nece-

31. Partimos de la base que el panel ya está constituido. Por eso obviamos mencionar la petición inicial de que se constituya un Dispute Board para entender en el caso. 
sario), el carácter de la intervención del Dispute Board y las pruebas que hagan a la posición de la parte ${ }^{32}$.

Una vez recibido el escrito, la parte contraria contará con un plazo específico para contestar dicho requerimiento de treinta días. Es común que los miembros del panel, al observar la controversia que se somete, puedan fijar otro plazo.

Creemos interesante poner de resalto una cuestión tratada por la Ley de PPP que podría aplicarse a otros países latinoamericanos. La Ley de PPP establece que no será necesario agotar ninguna vía administrativa o reclamo administrativo previo para iniciar el proceso de desavenencia frente al panel de expertos ${ }^{33}$. Esta determinación, que desde ya consideramos que es acertada, le brinda mayor seguridad al contratista que no vería demorado su reclamo por trámites burocráticos.

b. Luego de contestado el requerimiento, comienza la segunda etapa, de producción de prueba. Esta etapa es muy flexible, pudiendo contar o no con la existencia de pruebas a producirse, o con celebración de audiencias, visitas al sitio en donde se lleva a cabo el proyecto para analizar in situ el conflicto, entre otras medidas que pudiera adoptar el panel.

Así, estamos frente a un proceso dinámico, en donde deben primar ciertos principios esenciales: (i) las partes deben cooperar para resolver la controversia, siempre teniendo como norte el proyecto; (ii) prima la idea de transparencia y pleno acceso a la información por parte de los miembros del panel ${ }^{34}$; (iii) el panel de expertos conducirá el procedimiento de acuerdo al reglamento y a lo que considere más adecuado y conveniente para la resolución

32. El Art. 19 del Reglamento de Dispute Boards de la CCI contempla específicamente los puntos que debe contener el escrito de iniciación. Si bien los requisitos no parecieran ser exhaustivos, sí consideramos que son elementos básicos que cualquier parte debe tener presente a la hora de plantear la desavenencia a un panel de expertos. A diferencia de un arbitraje, no se prevé una instancia posterior para enfatizar la posición de cada una de las partes, por lo que el escrito de inicio debe ser lo más completo posible para generar la convicción en el panel de expertos de que la controversia debe solucionarse a su favor.

33. Art. 9.13.g).

34. Según CHERN esta intervención de los Dispute Boards es una de las razones por las cuales el instituto funciona. Véase, C. CHERN, N. 5, pp. 76 y ss. 
de la controversia y la finalidad del proyecto; y (iv) el panel de expertos tiene facultades conciliatorias más allá de las facultades para resolver la contienda.

Tal es la flexibilidad de esta etapa que los miembros del panel pueden dirigir el procedimiento según su mejor entender requiriendo nuevos escritos o pruebas ${ }^{35}$, o celebrando audiencias entre las partes para lograr (i) llegar a una solución amistosa; o (ii) que los miembros del panel tengan la posibilidad de escuchar a las partes, la presentación del caso y de las pruebas más relevantes ${ }^{36}$.

c. Terminada la etapa de prueba y demostración de la posición de cada parte, inclusive de las audiencias o visitas al proyecto, los miembros del panel de expertos deberán deliberar y dictar su resolución al respecto.

Recuérdese, las decisiones del panel de expertos podrán ser recomendaciones o decisiones vinculantes, dependiendo del tipo de panel de expertos que se adopte. Así, el Dispute Adjudication Board brindará una decisión vinculante para las partes, mientras que el Dispute Review Board emitirá una recomendación, que podrá tornarse vinculante en caso que así lo prevea el reglamento aplicable, si dentro de un plazo determinado, ninguna de las partes la impugnara por los mecanismos previstos.

Las decisiones deben ser tomadas por los miembros del panel de expertos lo más rápidamente posible. Respetándose siempre el principio de celeridad en las decisiones para hacer de este sistema una herramienta de resolución de controversias sin que afecte la normal ejecución del proyecto. A modo de ejemplo, el Reglamento de Dispute Boards de la CCI prevé que el plazo para dictar una decisión será determinado por el propio panel, pero nunca podrá exceder de noventa días contados desde la fecha de presentación de la controversia.

35. El Art. 15 del Reglamento de Dispute Boards de la CCI señala que son facultades del Dispute Board las de: (i) determinar el idioma del procedimiento; (ii) requerir que las partes aporten documentación; (iii) convocar a reuniones y audiencias; (iv) interrogar a las partes; (v) nombrar peritos; (vi) dictar remedios temporales, entre otras cuestiones.

36. C. CHERn, N. 5, pp. 73 y ss. 
A la hora de dictar la decisión (sin importar qué grado de vinculación tenga para las partes) el panel de expertos deberá ponderar: (i) los hechos del caso; (ii) los elementos aportados por las partes durante la ejecución del contrato, las audiencias celebradas para la resolución de la contienda, y las visitas al lugar; (iii) las disposiciones contractuales que ayudaron a los miembros del panel a tomar la decisión; (iv) determinar la forma de cumplimiento de la decisión; y, (v) explicar el razonamiento de la decisión.

Las decisiones incluyen, como requisito básico, la indicación de la fecha, la parte resolutiva y las razones que fundamentan la resolución ${ }^{37}$. También es necesario que la resolución cuente con un resumen de las posiciones de las partes, de las disposiciones del contrato, del procedimiento seguido y de los escritos y documentos presentados por las partes, conforme hemos mencionado anteriormente ${ }^{38}$.

En relación a la forma de tomar las decisiones, se pone de resalto que comúnmente las mismas son tomadas de manera unánime. Los miembros del panel deben buscar lograr dictar una decisión unánime, con la finalidad de generar certeza en las partes respecto de la decisión adoptada y de la forma de ejecutar el proyecto $^{39}$. Además, una decisión unánime tiene mayor fuerza a la hora de obligar su cumplimiento.

Para el caso de un panel de expertos celebrado en el marco de la CCI, la resolución tomada por el Dispute Board puede ser previamente examinada por el Centro Internacional de ADR de la Cámara de Comercio Internacional. Este examen será previo a la emisión formal de la decisión por parte del Dispute Board y solamente podrá corregirse la forma de la decisión ${ }^{40}$.

37. Véase, Art. 22 primer párrafo del Reglamento de Dispute Boards de la CCI.

38. Véase, Art. 22 segundo párrafo del Reglamento de Dispute Boards de la CCI.

39. Esta es la posición del Reglamento de Dispute Boards de la CCI que en el Art. 25 de dicho cuerpo normativo propone como primera opción.

40. Véase, Art. 23 del Reglamento de Dispute Boards de la CCI. 
d. Luego de emitida la decisión, será necesario proceder a su ejecución. La misma procederá de acuerdo al reglamento elegido, teniendo en consideración el tipo de decisión emitida y el tribunal jurisdiccional elegido por las partes.

En este sentido, las decisiones vinculantes emitidas por el Dispute Adjudication Board deberían ser de cumplimiento inmediato salvo que una de las partes presente una nota de disconformidad en los términos y en el plazo previsto por el reglamento aplicable.

En cuanto a las recomendaciones, las mismas serán vinculantes siempre y cuando no fueran impugnadas oportunamente por las partes y transcurriera el plazo legal establecido.

Por lo general, la elección de los Dispute Boards conlleva la elección de tribunales arbitrales, dada la experiencia en el tema de los posibles integrantes del tribunal, como así también la relación fluida que puede darse entre ambos sistemas. Las partes optarán por la administración de ambos mecanismos de resolución de controversias bajo una institución, pactando un reglamento que logre armonización entre ambos institutos.

Tal es el caso de la CCI. Esta institución posee un reglamento de Dispute Boards, tal como hemos visto, y también uno de arbitraje, que se relacionan de manera fluida.

Para el caso de la ejecución de una decisión del Dispute Board será necesario presentarla ante el tribunal arbitral, quien obligará a la parte a que cumpla con lo resuelto por el panel. Esta decisión luego puede ser presentada ante los tribunales judiciales para ser reconocida.

Dado que en la mayoría de los casos de ejecución de decisiones de los paneles de expertos se dan en la esfera de un arbitraje, no contamos con precedentes en la materia, de carácter público, que permitan llegar a una conclusión de cómo funciona el sistema. 
El único caso que ha cobrado publicidad por haber intervenido un tribunal judicial es el caso "Persero" ${ }^{41}$. En este antecedente, el panel de expertos resolvió que el comitente debía abonar la suma de US\$17.000.000 al contratista. El comitente presentó dentro del plazo legal permitido, una nota de disconformidad con la decisión del Dispute Adjudication Board y se negó a pagar. El contratista inició un arbitraje contra el comitente para obtener el pago de la suma adeudada en el año 2009.

El tribunal arbitral dictó un laudo que fue dejado sin efecto por el Tribunal Superior de Singapur y la Cámara de Apelaciones de dicho país, con el argumento de que el laudo estuvo mal dictado ya que un laudo final ejecutando una decisión de un panel de expertos no permitiría discutir los méritos de la decisión. En consecuencia, ambos tribunales judiciales entendieron que el tribunal arbitral debió haber dictado una medida cautelar o un laudo parcial sobre este tema.

En el año 2011, el contratista inició un nuevo arbitraje con un nuevo tribunal con la finalidad de ejecutar la decisión del panel de expertos. Allí, el contratista obtuvo un laudo preliminar ordenando el pago de las sumas adeudadas por el comitente. El contratista inició el procedimiento de reconocimiento y ejecución del laudo frente al Tribunal Superior de Singapur, mientras que el comitente inició frente al mismo tribunal, el proceso de anulación del laudo.

En primera instancia, el tribunal decidió ordenar la ejecución del laudo. El comitente apeló la medida frente a la Cámara de Apelaciones de Singapur, quien confirmó la decisión de primera instancia. La Cámara sostuvo que el laudo no afectaba el derecho del comitente de reclamar la revisión total de los méritos de la decisión del panel de expertos ${ }^{42}$.

41. Cámara de Apelaciones de Singapur, PT Perusahaan Gas Negara (Persero) TBK v. CRW Joint Operation, (2011 y 2015).

42. Véase con mayor detalle, J. Wright, "Enforcement of interim binding Dispute Board decisions", «https://goo.gl/eP8pCC», (01/06/15). 
e. Finalmente, debe considerarse el proceso de impugnación de una decisión de un panel de expertos. Hemos efectuado algunas manifestaciones al respecto en el punto $\mathbf{d}$. anteriormente desarrollado. En especial, nos hemos referido a este supuesto cuando tratamos el caso Persero.

Sin perjuicio de ello, haremos un análisis más completo sobre los mecanismos que tienen las partes para impugnar las decisiones, con las consecuencias sobre las mismas. Para este análisis, partiremos de la base de que las partes eligieron el arbitraje como mecanismo jurisdiccional de resolución de controversias, ya que eso nos permitirá estudiar con mayor precisión su interacción con el panel de expertos.

Las decisiones de los paneles de expertos pueden ser revisadas en su totalidad. Las partes pueden impugnar dichas revisiones con base en los mecanismos previstos en el reglamento aplicable. Esas impugnaciones se presentarán frente a los tribunales elegidos por las partes, y con las exigencias determinadas por el reglamento a aplicar.

Así, para el caso de elegirse un tribunal arbitral, la parte perjudicada por la decisión del panel de expertos presentará su reclamo para revisar la decisión y los méritos del caso. Esta presentación no impedirá que continúe la ejecución de la medida por vía arbitral.

Como hemos visto, luego se podrá solicitar la nulidad del laudo que ordena ejecutar la medida adoptada por el panel de expertos. También podrá reclamarse los daños y perjuicios que la medida cause, si es que con posterioridad un tribunal arbitral resolvió que la decisión del panel es errónea. 


\section{Conclusiones}

A manera de cierre, podemos concluir que los paneles de expertos o Dispute Boards presentan grandes ventajas para las partes a la hora de ejecutar contratos de construcción. Estos paneles colaborarán activamente en el desarrollo del proyecto conforme fuera estipulado y, más importante aún, dentro del plazo fijado originariamente.

La experiencia global ha demostrado que los paneles de expertos son mecanismos eficientes y aptos para resolver conflictos en el marco de contratos de obra. Así, puede observarse que en contratos de obra donde se pactaron Dispute Boards el proyecto se ejecutó en menor tiempo. También han ayudado a un mejor control por parte del dueño de la obra y a evitar gastos y costos innecesarios por la demora en la ejecución.

Estamos en presencia de un sistema que puede implementarse en el derecho latinoamericano en general, y en el derecho argentino en particular. Ordenamientos jurídicos de la región ya han incorporado este sistema de resolución de disputas. Ahora resta observar si esta herramienta podrá ser aplicada eficazmente.

Creemos que este desafío -el correcto uso de los Dispute Boards- puede ser sorteado con éxito en nuestra región dado los pocos ejemplos que pudimos observar en los últimos años utilizando el sistema de manera eficaz y precisa. Será luego tarea de los practicantes fomentar el uso de este mecanismo, como así también del Estado, posibilitando su inclusión en obras de envergadura. 